\title{
A RECEPCฺÃO DA EPISTEMOLOGIA DE FLECK PELA PESQUISA EM EDUCAÇÃO EM CIÊNCIAS NO BRASIL
}

\author{
Leonir Lorenzetti* \\ Cristiane Muenchen** \\ lône Inês Pinsson Slongo***
}

RESUMO: Este artigo apresenta um estudo que investigou a recepção da epistemologia de Ludwik Fleck pela pesquisa em Educação em Ciências. Foram analisadas teses e dissertações produzidas no período de 1995 a 2010, em programas nacionais de pós-graduação. Os dados apontam que na década de 1990 surgiram os primeiros estudos e que há uma concentração de trabalhos em instituições do Sul do Brasil, notadamente na UFSC, especialmente na área da Educação em Ciências, estando o maior volume de estudos concentrados nos eixos "emergência de um fato científico", "formação de professores" e "análise da produção acadêmica". Destaca-se a significativa contribuição das categorias epistemológicas "estilo de pensamento", "coletivo de pensamento" e "circulação intra e intercoletiva de ideias" no processo de produção do conhecimento.

Palavras-chave: Ludwik Fleck; Produção Acadêmica; Educação em Ciências.

\section{THE RECEPTION OF THE EPISTEMOLOGY OF FLECK FOR THE RESEARCH IN SCIENCE EDUCATION}

ABSTRACT: This article presents a study that investigated the reception of the epistemology of Ludwik Fleck for the inquiry in Science Education. Theses and dissertations produced in the period from 1995 to 2010, in national programs of post graduation, were analyzed. The data point that the first studies appeared in the decade of 1990 and that they were concentrated in institutions of the Southern Brazil, particularly in UFSC, especially in the area of the Science Education. The largest volume of studies are concentrated around the themes "emergence of a scientific fact", "teachers' formation" and "analysis of the academic production". It stands out that there was a significant contribution of the epistemological categories "style of thought", "collective of thought" and "circulation of intra and inter-collective ideas" in the process of knowledge production.

Keywords: Ludwik Fleck; Academic Production, Education in Science. 


\section{INTRODUÇÃO E REFERENCIAL TEÓRICO}

Este artigo relata o estudo que investigou a recepção da epistemologia de Fleck $(1986 ; 2010)$ pela pesquisa em Educação em Ciências desenvolvida no Brasil. Foram analisadas teses e dissertações produzidas pela área no período entre 1995 e 2010, as quais tiveram como referência a abordagem epistemológica desse autor.

Ludwik Fleck (1896-1961), médico e filósofo de origem judia, nasceu em 1896, em Lwów, na Polônia, atual região da Ucrânia, e morreu em Israel, em 1961. Dedicou-se à medicina, atuando como clínico e pesquisador nas áreas de bacteriologia, microbiologia e imunologia, tendo sido um dos precursores desta última. Paralelamente, estudou sociologia, filosofia e história da ciência (SCHÄFER; SCHNELLE, 1986), acumulando importante produção no campo da epistemologia (DA ROS, 2000; DELIZOICOV et al., 2002; PFUETZENREITER, 2003).

Influenciado pela Escola Polonesa de Filosofia da Medicina e contrapondo-se ao empirismo lógico do Círculo de Viena (LÖWY, 2004), Fleck desenvolveu sua reflexão epistemológica, partindo da premissa de que o conhecimento é fruto de processos sócio-históricos, efetuado por coletivos de pensamento em interação sociocultural. Considerou que o conhecimento produzido por esses coletivos está em conformidade com uma estrutura de pensamento predominante na sociedade em cada momento histórico. Desse modo, Fleck $(1986 ; 2010)$ propôs as categorias epistemológicas estilo de pensamento, coletivo de pensamento, circulação intercoletiva e intracoletiva de ideias, com as quais analisou a gênese e a difusão de conhecimentos e práticas produzidas por esses coletivos.

Ao descrever a trajetória histórica do conceito de sífilis (FLECK, 1986; 2010), definiu coletivo de pensamento como sendo a unidade social da comunidade de cientistas de um campo determinado do saber e estilo de pensamento como sendo o conjunto de pressuposições sobre as quais o coletivo de pensamento constrói seu edifício teórico. Argumentou que o saber nunca é possível em si mesmo, mas sob determinadas condições e suposições sobre o objeto as quais não podem tornar-se compreensíveis a priori, mas como produto histórico e sociológico da atuação de um coletivo de pensamento. Desse modo, caracterizou estilo de pensamento como o conjunto de conhecimentos e práticas, concepções, tradições e normas compartilhadas pelos membros do coletivo de pensamento, cabendo-lhe o papel de direcionador do modo de pensar e de agir do coletivo de pensamento, possibilitando-lhe uma maneira própria de ver e interagir com o objeto do conhecimento. Por sua vez, sendo o coletivo de pensamento o portador comunitário do estilo de pensamento, cabe-lhe determinar, em cada época, os problemas de pesquisa pertinentes e relevantes a serem solucionados (FLECK, 1986; 2010).

$\mathrm{Na}$ estrutura geral do coletivo de pensamento, Fleck $(1986 ; 2010)$ distinguiu os círculos esotérico e exotérico. Segundo o autor, o círculo esotérico é formado pelos especialistas de uma área do conhecimento e o círculo exotérico, pelos leigos e leigos formados. As pessoas podem pertencer a vários coletivos simultaneamente, atuando como veículos na transmissão de ideias entre eles. 
A presença de um círculo esotérico, formado por especialistas de uma determinada área do conhecimento, caracteriza a identidade primeira do coletivo de pensamento, por ser este o portador do estilo de pensamento. É a partir desse núcleo de conhecimentos e de práticas compartilhadas que se formam os círculos exotéricos, quando passam a interagir, por meio de múltiplas alternativas, com o círculo esotérico. Entre os círculos esotérico e exotérico estabelecem-se relações dinâmicas que contribuem para a ampliação da área de conhecimento, denominadas de circulação intracoletiva e intercoletiva de conhecimentos e práticas.

A circulação intracoletiva ocorre no interior do coletivo de pensamento, assegurando a extensão do estilo de pensamento, bem como o compartilhamento dos conhecimentos e práticas relativas ao estilo de pensamento vigente, de modo a formar os novos membros do grupo (FLECK, 1986; 2010). Por sua vez, a circulação intercoletiva de ideias ocorre entre dois ou mais coletivos de pensamento, contribuindo, de modo significativo, com a transformação do estilo de pensamento, pois "[...] qualquer tráfego intercoletivo de pensamento traz consigo um deslocamento ou uma alteração dos valores de pensamento" (FLECK, 2010, p. 161).

$\mathrm{O}$ autor considera, ainda, que a relação cognoscitiva entre sujeito e objeto a conhecer é mediatizada por um terceiro fator, o estado do conhecimento, que pressupõe as relações históricas, sociais e culturais. Assim sendo, as relações históricas presentes em um determinado estilo de pensamento indicam uma inter-relação entre o conhecido e o que se quer conhecer. Logo, o processo de produção de conhecimento deve levar em consideração três elementos: o sujeito, o objeto e o estilo de pensamento compartilhado pelo coletivo de pensamento.

Na reconstrução histórica do conceito de sífilis, Fleck (1986; 2010) observou uma dinâmica que se repete na "marcha" do pensamento, fazendo com que o modo de ver, pensar e agir, ao mesmo tempo em que resiste, sofre transformações. A essa dinâmica denominou de instauração, extensão e transformação dos estilos de pensamento. Argumentou que as teorias científicas vivem, primeiro, uma fase clássica, quando todas as ideias estão em conformidade com o pensamento vigente ou com a teoria dominante. Nessa fase, o estilo de pensamento acha-se devidamente instaurado, e o esforço do coletivo de pensamento é no sentido de desenvolver o pensamento dominante, fase denominada por Fleck (1986; 2010) de extensão do estilo de pensamento. Nesse momento, verifica-se uma ampliação do rol de problemas a investigar. Contudo, o autor aponta que, apesar da tendência à persistência, há um momento em que as "complicações" aparecem, ou seja, surgem as exceções, os problemas que o estilo de pensamento não consegue resolver. Assim, instala-se um período de instabilidade e controvérsias, intensificando o debate intra e intercoletivos de pensamento. Significativas mudanças marcam esse período, as quais determinam o que Fleck $(1986 ; 2010)$ denomina de transformação do estilo de pensamento e que culmina com a emergência de um novo modo de pensar e agir, isto é, um novo estilo de pensamento.

Em síntese, o pensamento epistemológico de Fleck (1986; 2010) introduz parâmetros de análise sobre o processo de produção e disseminação do conhecimento, 
estabelecendo e caracterizando as categorias que balizam sua epistemologia, com destaque para estilo de pensamento, coletivo de pensamento, circulação intercoletiva e intracoletiva de ideias, instauração, extensão e transformação do estilo de pensamento. Essas categorias epistemológicas vêm sendo utilizadas de modo recorrente e crescente no país, de forma significativa pela pesquisa em Educação em Ciências (DELIZOICOV et al., 2002; SLONGO, 2004; LORENZETTI, 2008 e MUENCHEN, 2010).

Este artigo apresenta, inicialmente, um panorama das pesquisas que utilizam o aporte epistemológico de Fleck, com destaque para a produção em Educação em Ciências, procurando explicitar em que momento ele se estabeleceu como referencial para a pesquisa na área, os objetos/programas/pesquisadores que buscaram esse diálogo epistemológico, a justificativa apontada para a utilização dessa epistemologia e as contribuições que a abordagem trouxe à pesquisa na área, segundo os autores dessas pesquisas.

\section{PROCEDIMENTOS METODOLÓGICOS E PANORAMA INICIAL}

$\mathrm{Na}$ atualidade, os estudos do tipo "estado do conhecimento" (FERREIRA, 2002) são realizados nas diferentes áreas do conhecimento, com significativa contribuição para proporcionar a divulgação e o intercâmbio do conhecimento já produzido pela área, tendo em vista os desafios atuais e, desse modo, otimizar a pesquisa em diversos aspectos.

O estudo aqui relatado configurou-se como pesquisa bibliográfica, do tipo "estado do conhecimento", e teve o objetivo de analisar a presença e a recepção da epistemologia de Ludwik Fleck (1986; 2010) na pesquisa em Educação em Ciências. Desta forma, foram analisadas dissertações e teses desenvolvidas em programas nacionais de pós-graduação, no período de 1995 a 2010, e que utilizaram a epistemologia de Fleck como referencial teórico.

Os dados foram coletados no primeiro semestre de 2011. Inicialmente, foi realizado um mapeamento de dissertações e teses orientadas pela matriz epistemológica de Fleck no banco de teses da CAPES, no sítio virtual http://www.capes. gov.br/servicos/banco-de-teses. Para a localização dos trabalhos, foram utilizadas as seguintes expressões exatas "Ludwik Fleck", "Fleck" e "Estilo de Pensamento", por meio das quais foi possível localizar 40 pesquisas, envolvendo dissertações e teses.

Posteriormente, foram analisadas quatro teses desenvolvidas na Universidade Federal de Santa Catarina, nos programas de Educação (DA ROS, 2000 e SLONGO, 2004) e Educação Científica e Tecnológica (LORENZETTI, 2008 e MUENCHEN, 2010). Tais pesquisas utilizaram fortemente o referencial fleckiano e, ao fazê-lo, resgataram contribuições de estudos realizados anteriormente e que estiveram pautados nessa matriz epistemológica. Assim, ao analisar essas quatro teses, foi possível identificar 25 estudos desenvolvidos em programas nacionais de pós-graduação, nas áreas de Educação em Ciências, Filosofia da Ciência e Saúde, os quais passaram a integrar a amostra da presente investigação. 
Com esse levantamento, verificou-se que 24 das pesquisas mapeadas nos trabalhos de Da Ros (2000), Slongo (2004), Lorenzetti (2008) e Muenchem (2010) estavam contempladas no banco de teses da Capes.

Desta forma, a amostra foi constituída por 41 trabalhos que utilizam a epistemologia de Fleck como referencial teórico. Na sequência, uma leitura cuidadosa do resumo de cada dissertação e tese foi realizada, objetivando verificar a pertinência e a vinculação do trabalho com o presente estudo.

Vale ressaltar que as pesquisas de Da Ros (2000), Slongo (2004), Lorenzetti (2008) e Muenchen (2010), além de contribuírem para a identificação dos textos que seriam analisados, constituíram-se como referências fundamentais para a realização deste estudo, orientando os elementos a serem identificados nas pesquisas objeto de análise desta investigação.

Em um a primeira aproximação, as pesquisas mapeadas geraram tabelas que identificaram volume de trabalhos desenvolvidos no período e concentração de trabalhos por regiões, áreas do conhecimento, IES, programas e respectivos orientadores.

Pelo levantamento realizado, foi possível identificar que a epistemologia de Fleck passou a orientar os primeiros estudos em meados da década de 1990, tendo, até 2010, subsidiado o desenvolvimento de 41 estudos, sendo 23 dissertações e 18 teses, uma relação praticamente equilibrada entre ambas as modalidades, assim distribuídas ao longo do período:

Tabela 1: Número de trabalhos por período

\begin{tabular}{|c|c|c|c|}
\hline Período & Dissertações & Teses & Total \\
\hline 10 Período (1995 a 2000) & 4 & 2 & 6 \\
\hline $2^{\circ}$ Período (2001 a 2005) & 6 & 10 & 16 \\
\hline $3^{\circ}$ Período (2006 a 2010) & 13 & 6 & 19 \\
\hline Total & 23 & 18 & 41 \\
\hline
\end{tabular}

A partir da leitura dos resumos, foi possível identificar a área do conhecimento de abrangência desses trabalhos, classificando-os em Saúde, Educação em Ciências e Filosofia da Ciência. Conforme Tabela 2, fica evidente a concentração de trabalhos nas áreas de Saúde e de Educação em Ciências.

Tabela 2: Número de trabalhos por área do conhecimento

\begin{tabular}{|c|c|}
\hline Área de Conhecimento & Total \\
\hline Saúde & 23 \\
\hline Educação em Ciências & 13 \\
\hline Filosofia da Ciência & $\mathbf{5}$ \\
\hline Total & 41 \\
\hline
\end{tabular}


Em termos geográficos, conforme Tabela 3, observa-se que a recepção de Fleck está fortemente concentrada em programas da região Sul do Brasil, com trinta trabalhos defendidos no período investigado, especialmente no estado de Santa Catarina, seguida pela região Sudeste, nos estados do Rio de Janeiro, Minas Gerais e São Paulo, com nove trabalhos, e pela região Centro-Oeste, no Distrito Federal, com dois trabalhos.

Tabela 3: Número de trabalhos por região e Estado

\begin{tabular}{|c|c|c|}
\hline \multirow{2}{*}{ Região } & Estados & Total \\
\hline \multirow{3}{*}{ Sul } & SC & $\mathbf{2 8}$ \\
\cline { 2 - 3 } & PR & $\mathbf{1}$ \\
\cline { 2 - 3 } & RS & $\mathbf{1}$ \\
\hline \multirow{3}{*}{ Sudeste } & RJ & 6 \\
\cline { 2 - 3 } & MG & 2 \\
\cline { 2 - 3 } & SP & 1 \\
\hline Centro-Oeste & DF & 2 \\
\hline \multicolumn{2}{|c}{ Total } & 41 \\
\hline
\end{tabular}

Tabela 4: Número de trabalhos por programa e IES

\begin{tabular}{|c|c|c|}
\hline Programa & IES & Total \\
\hline Educação & UFSC, UFPR, UNESC e UnB & $\mathbf{1 5}$ \\
\hline Saúde Coletiva & $\begin{array}{c}\text { UFSC, UERJ, FUNDAÇÃO } \\
\text { OSWALDO CRUZ, UNICAMP } \\
\text { e UFRJ }\end{array}$ & $\mathbf{7}$ \\
\hline $\begin{array}{c}\text { Educação Científica } \\
\text { e Tecnológica }\end{array}$ & UFSC \\
\hline Saúde & UNIVALE & $\mathbf{6 4}$ \\
\hline Filosofia & UFSC e UERJ & $\mathbf{2}$ \\
\hline História & UFMG & $\mathbf{6}$ \\
\hline Outros* & UNIJUÍ e UFSC & $\mathbf{4 1}$ \\
\hline \multicolumn{2}{|c|}{ Total }
\end{tabular}

*Educação Física, Educação nas Ciências, Enfermagem, Engenharia Ambiental, Sociologia Política e Saúde Pública.

Cruzando os dados das Tabelas 2 e 4, observa-se que embora a área de Saúde lidere em número de trabalhos subsidiados pela matriz epistemológica de Fleck, mais de 50\% desses estudos foram desenvolvidos em outros programas, como é possível identificar na Tabela 4. Esse dado exemplifica muito bem a circulação intercoletiva de ideias no processo de produção do conhecimento, conforme 
argumentou Fleck (1986; 2010). Exemplificam essa importante dinâmica as teses de Da Ros (2000), Cutolo (2001) e Pfuetzenreiter (2003), as quais, mesmo focando em objetos específicos da área de Saúde, foram geradas no Programa de Pós-Graduação em Educação da UFSC.

Levantamento realizado por Lorenzetti (2008) indica a existência de centros de estudos no Brasil que utilizam Fleck como referência, mostrando que a citação de sua obra em pesquisas nacionais apresentam uma trajetória e uma aplicação em diferentes contextos. Esses centros estão localizados na Universidade Federal de Santa Catarina, envolvendo os programas de Educação, Enfermagem, Saúde Pública, Filosofia, Sociologia Política e Educação Científica e Tecnológica. No estado do Rio de Janeiro, a produção envolvendo a epistemologia de Fleck está concentrada no programa de Saúde Coletiva da Fundação Oswaldo Cruz e nos programas de Saúde Coletiva e Filosofia da Universidade do Estado do Rio de Janeiro. Já na Universidade Federal de Minas, a epistemologia de Fleck foi utilizada no programa de História.

Neste estudo, constata-se que a epistemologia de Fleck está sendo utilizada em outras universidades, tais como UNIVALI, UFPR, UNICAMP, UNESC, UNIJUÍ e UnB, o que demonstra a disseminação da epistemologia de Fleck na comunidade acadêmica, conforme Tabela 4.

Com relação aos orientadores das dissertações e teses, os dados indicam a liderança de Demétrio Delizoicov, que orientou oito estudos; Edel Ern e Luiz Roberto Agea Cutolo, quatro estudos cada; Kenneth Rochel de Camargo Jr., três estudos; Arden Zylbersztajn, Maria Helena da Silva Carneiro, Mauro Lúcio Leitão Conde e Nadir Ferrari, dois estudos cada; Alberto Oscar Cupani, Araci Asinelli da Luz, Carlos Alberto Marques, Charles Dalcanale Tesser, Edgard Matiello Júnior, Elena Moraes Garcia, Elizabeth Farias da Silva, Elizabeth Moreira dos Santos, Ilton Benoni da Silva, Jeni Vaitsman, Madel Therezinha Luz, Marco Aurélio da Ros, Maria Cristina Pansera-de-Araújo e Sergio Roberto Martins, um estudo cada.

Os dados mostram que, apesar da relativa dispersão dos trabalhos por IES, programas ou orientadores, os estudos pautados na epistemologia fleckiana têm se mantido regulares ao longo do período analisado e com tendência à ascensão.

Queirós e Nardi (2008) apresentam um panorama da produção acadêmica que utiliza a epistemologia de Fleck como referencial teórico e que foi publicada em periódicos nacionais da área de Educação em Ciências e nas atas do Encontro Nacional de Pesquisa em Educação em Ciências, realizado no período de 2002 a 2007. Com esse foco, esses autores localizaram 13 artigos e apontaram o predomínio de objetos vinculados à área de Saúde e Ciências Biológicas. Além disso, classificaram esses estudos em quatro grandes categorias: 1) História de Fatos Científicos; 2) Concepção de professores acerca da natureza da ciência; 3) Ensino de Saúde; 4) Análises de pesquisas em Ensino de Ciências no Brasil.

De outro vértice, o estudo de Lorenzetti (2008) localizou 20 pesquisas, envolvendo dissertações e teses desenvolvidas em programas de pós-graduação, no período de 1995 a 2006. Essa produção acadêmica, que se apoia nas ideias 
epistemológicas de Fleck, foi categorizada pelo autor em cinco eixos: 1) Formação de professores; 2) Estudos sobre o currículo; 3) Análise sobre a emergência de um fato; 4) Relação de Fleck com outros autores; 5) Análise de produção acadêmica.

Considerando a relevância dessa classificação, na medida em que melhor explicita a natureza dos objetos investigados à luz da epistemologia fleckiana, ela foi utilizada na análise das 41 teses e dissertações que têm como referencial a epistemologia de Fleck (1986; 2010), conforme análise que segue:

- Formação de professores: a categoria de Estilo de Pensamento de Fleck foi utilizada em quatro dissertações de mestrado e uma tese de doutorado para analisar o trabalho pedagógico docente, a partir de uma investigação das práticas efetivadas (DELIZOICOV, 1995; LIMA, 1999; LAMBACH, 2007; LÜDKE, 2009 e MUENCHEN, 2010);

- Estudos sobre o currículo: a epistemologia de Fleck foi empregada em dez trabalhos para analisar o currículo escolar, principalmente nos cursos relacionados à área de Saúde (AMORIM, 2004; BACKES, 1999; CUTOLO, 2001; KOIFMAN, 1996; NOGUEIRA, 2003; LIMA, 2003; PFUETZENREITER, 2003; MAEYAMA, 2006; SANTOS, 2005 e BISCHOFF, 2009);

- Análise sobre a emergência de um fato científico: as discussões sobre o surgimento de determinados fatos, o processo de produção de conhecimento sobre estes, envolvendo a utilização das categorias de instauração, extensão e transformação de Estilo de Pensamento e o papel da circulação intracoletiva e intercoletiva de Fleck foram realizadas em dezessete trabalhos (ARAÚJO, 2002; GOMES, 2002; DELIZOICOV, 2002; LEITE, 2004; QUARESMA, 2005; SANTOS, 1999; UCHÔA, 2003; SCHEID, 2006; BERTONI, 2007; COSTA, 2009; GUEDES, 2007; MACHADO, 2008; SILVA, 2009; SKALINSKI, 2008; TESSER, 2004; LIMA, 2007 e TAVARES, 2008);

- Relação de Fleck com outros autores: nesse eixo, foram classificadas quatro dissertações que utilizam Fleck como referência, estabelecendo relações com outros epistemólogos, na forma de análise da obra de Fleck e seus reflexos sobre a produção do conhecimento, principalmente a influência exercida na obra de Thomas Kuhn, intitulada "Estrutura das Revoluções Científicas” (NEDER, 2001; KOSLOWSKI, 2004; PARREIRAS, 2001 e SALLES, 2007);

- Análise de produção acadêmica: foram localizados cinco trabalhos que analisam produção científica em determinada área do conhecimento a partir de dissertações e teses defendidas em programas de pós-graduação (DA ROS, 2000; SLONGO, 2004; LORENZET'TI, 2008; MEZALIRA, 2008 e CAVALLI, 2009).

Observa-se que dois eixos aglutinam aproximadamente $70 \%$ dos trabalhos analisados: emergência de um fato científico com 17 trabalhos, e estudos sobre currículo com dez. Os demais eixos apresentam uma produção relativamente equilibrada. 
Após esse levantamento e análise inicial das 41 teses e dissertações que utilizaram o aporte epistemológico de Fleck (1986; 2010), um estudo específico foi realizado envolvendo 11 das 13 pesquisas, cujas problemáticas investigadas pertencem à grande área de Educação em Ciências. Vale destacar que dois documentos não foram analisados por não estarem disponíveis na ocasião em que o estudo foi realizado.

Buscaram-se os seguintes elementos, utilizando a metodologia de análise de conteúdo (BARDIN, 2008), principalmente no resumo, introdução, metodologia, considerações finais e referências bibliográficas dos textos das teses e dissertações: foco temático, problema de pesquisa, justificativas apontadas pelo autor para utilizar a epistemologia de Fleck, categorias fleckianas utilizadas, referências às obras de Fleck e às pesquisas que utilizaram Fleck e contribuições do referencial epistemológico para a pesquisa em Educação em Ciências, apontadas pelo estudo. Esses dados e sua respectiva análise encontram-se a seguir.

\section{A EPISTEMOLOGIA DE FLECK E A PESOUISA EM EDUCAC̦ÃO EM CIÊNCIAS}

Das 11 pesquisas analisadas, 7 são teses e 4 são dissertações. Os dados estão na Tabela 5 .

Tabela 5: Volume de pesquisas por IES/Programa

\begin{tabular}{|c|c|c|c|c|}
\hline IES & Programa & Dissertações & Teses & Total \\
\hline \multirow{3}{*}{ UFSC } & Educação & 1 & 3 & 4 \\
\hline & Educação Científica e Tecnológica & 1 & 3 & 4 \\
\hline & Engenharia Ambiental & & 1 & 1 \\
\hline UNIJUÍ & Educação nas Ciências & 1 & & 1 \\
\hline UFPR & Educação & 1 & & 1 \\
\hline \multicolumn{2}{|r|}{ Total } & 4 & 7 & 11 \\
\hline
\end{tabular}

Considerando a categorização realizada por Lorenzetti (2008), constatou-se que das 11 pesquisas que abarcam a área de conhecimento Educação em Ciências, 5 estudos englobam o eixo Emergência de um fato cientifico, 3 discutem Formação de professores e 3 envolvem análises sobre a Produção acadêmica.

Ao considerar a área de conteúdo das 11 pesquisas analisadas, identificou-se que 5 trabalhos envolvem Biologia, 3 discutem Ensino de Ciências, 2 enfocam Educação Ambiental e 1 analisa Química. Esse dado está em sintonia com a pesquisa desenvolvida por Queiros e Nardi (2008) que, ao analisar a produção da área pautada na epistemologia de Fleck e publicada em periódicos nacionais e nas atas do ENPEC, constata o predomínio de trabalhos na área de Ciências Biológicas e Saúde. 
Seguindo a categorização proposta por Lorenzetti (2008), identificou-se que 11 estudos, cujos objetos investigados pertencem à grande área Educação em Ciências, ficaram distribuídos em três eixos: Emergência de um fato científico (cinco estudos), Formação de professores (três) e Análise da produção acadêmica (três).

Foram classificados no eixo Emergência de um fato científico os seguintes estudos: Leite (2004), Delizoicov, (2002), Scheid (2006), Lima (2007) e Bertoni (2007).

A tese de Leite (2004) identificou e analisou a visão de ciência e a dimensão histórica presente nos livros de genética utilizados no curso de Ciências Biológicas da UFSC. Uma análise epistemológica de um episódio histórico (caso de Mendel) foi realizada para contrapor e superar a visão empirista predominante nos livros e na educação científica em geral. O estudo de Delizoicov (2002) analisou o conhecimento sobre o movimento do sangue no corpo humano, considerando os contextos de sua produção e de sua disseminação. Scheid (2006) investigou aspectos epistemológicos presentes na história de um fato científico e as contribuições proporcionadas pela discussão desses aspectos no processo de ensino e de aprendizagem da Biologia, particularmente da Genética, e na melhoria da formação inicial de professores de Ciências Biológicas. Lima (2007) analisou o processo de planejamento e implantação do Parque Natural Municipal de Lages - SC, com ênfase na Conservação dos Recursos Hídricos e na percepção da comunidade. Bertoni (2007) identificou e fundamentou estilos de pensamento biológico que historicamente predominaram no modo de interpretar e compreender o fenômeno vida, objeto de estudo da Biologia.

Esses estudos, em seus distintos recortes, fazem um resgate e uma análise histórico-epistemológica do processo de produção de determinado conhecimento. Ao realizar esse percurso, esses autores explicitam Estilos e Coletivos de Pensamento, especialmente os estudos de Leite (2004) e Scheid (2006), que adicionam a esse foco o desafio de identificar, na trajetória de produção do conhecimento, elementos que possibilitam aos professores em formação uma compreensão mais adequada sobre a natureza da ciência.

No eixo Formação de professores, foram aglutinados os estudos dos seguintes autores: Delizoicov (1995), Marques (2007) e Muenchen (2010). Essas pesquisas buscam, cada uma, a seu modo, identificar Estilos de Pensamento a partir da análise de suas concepções educacionais e de suas práticas pedagógicas. Assim, Delizoicov (1995) identifica Estilos de Pensamento dos professores de Ciências do Ensino Fundamental, ao analisar sua interação com os livros didáticos; Marques (2007) identifica Estilos de Pensamento dos professores de Química que atuam em programas de Educação de Jovens e Adultos em escolas públicas do Paraná; por fim, o estudo de Muenchen (2010) busca caracterizar os processos investigativos que culminaram na proposição de uma estrutura didático-pedagógica ao Ensino de Ciências denominada de "Três Momentos Pedagógicos" (DELIZOICOV; ANGOTTI; PERNAMBUCO, 2002). Investigou-se, também, o processo de disseminação dessa dinâmica por um grupo de docentes da Universidade Federal de Santa Maria que atua na formação de professores de Ciências. Ao realizar esse 
percurso, o estudo utiliza intensamente as categorias circulação intracoletiva e circulação intercoletiva de ideias.

No eixo Análise da produção acadêmica foram aglutinados os estudos de Slongo (2004), Lorenzetti (2008) e Mezalira (2008), que analisaram, respectivamente, a produção em ensino de Biologia, Educação Ambiental e Ciência, Tecnologia e Sociedade e em ensino de Ciências Naturais. Tais pesquisas caracterizam-se como estudos histórico-epistemológicos que, ao analisar a produção acadêmica nas respectivas áreas de conhecimento e específicos recortes temporais, identificam Estilos e Coletivos de Pensamento. Isso acontece principalmente nos estudos de Slongo (2004) e Lorenzetti (2008) que, ao explicitarem os Estilos e Coletivos de Pensamento, descrevem seu o processo de instauração, extensão e transformação. Nesse sentido, em sintonia com o estudo de Muenchen (2010), as categorias de circulação intracoletiva e intercoletiva de ideias foram amplamente utilizadas.

Conforme já destacado, para Fleck (1986; 2010) o conhecimento deriva de uma interação entre o sujeito e o objeto, intercedida por uma dimensão que é social e culturalmente determinada. Nesse sentido, ele propõe que o processo de produção de conhecimento se concretiza nessa interação do sujeito com o objeto, mediado pelo que denomina de Estilo de Pensamento e no interior de um Coletivo de Pensamento.

Todos os estudos analisados utilizaram a categoria Estilo de Pensamento em suas análises. Assim, foi possível perceber que seu processo de produção de conhecimento levou em consideração os três elementos destacados por Fleck (1986; 2010): o sujeito, o objeto e o estilo de pensamento compartilhado pelo coletivo de pensamento.

De acordo com Fleck (1986; 2010), a produção do conhecimento caracteriza-se como um processo que envolve instauração, extensão e transformação de estilos de pensamento. $\mathrm{O}$ processo de instauração de um estilo de pensamento decorre do enfrentamento de um problema por mais de um pesquisador, ou seja, por um coletivo. É importante enfatizar que a identificação de um particular estilo de pensamento, bem como suas possíveis transformações se faz com a contribuição de resgates históricos, conforme argumentação de Fleck (1986; 2010). Esse aspecto é caracterizado de modo pertinente na tese de Scheid (2006), no que diz respeito ao caso da proposição da estrutura do DNA, e na de Delizoicov (2002), que estuda os modelos para o movimento do sangue no corpo humano propostos por Harvey e Cláudio Galeno. Com relação ao papel do contexto histórico, destaca-se também o trabalho de Leite (2004), que identificou e analisou a visão de ciência e a dimensão histórica presente nos livros de genética utilizados no curso de Ciências Biológicas da UFSC.

A perspectiva fleckiana também tem fundamentado análises da produção contemporânea. Algumas pesquisas, como as destacadas a seguir, abarcam análises de aspectos relativamente contemporâneos se comparados com a produção do conhecimento que foi objeto das análises histórico-epistemológicas de episódios que já fazem parte da História da Ciência, como os estudos sobre o modelo de Harvey referente à circulação sanguínea (DELIZOICOV; 2002), sobre as Leis de 
Mendel (LEITE, 2004) e sobre o DNA (SCHEID, 2006). Foram identificadas três teses de doutorado: Slongo (2004), Lorenzetti (2008) e Muenchen (2010), e quatro dissertações de mestrado: Delizoicov (1995), Bertoni (2007), Lambach (2007) e Mezalira (2008). As teses foram desenvolvidas na Universidade Federal de Santa Catarina. O estudo de Slongo (2004) enfoca a área de ensino de Biologia, o de Lorenzetti (2008) a área de Educação Ambiental e o de Muenchen (2010) a dinâmica dos Três Momentos Pedagógicos na área de ensino de Ciências. Já as dissertações de mestrado, duas delas desenvolvidas na Universidade Federal de Santa Catarina, enfocam, respectivamente, o livro didático de Ciências Naturais, o pensamento biológico a partir de Diretrizes Curriculares e as práticas de professores de Química da EJA do estado do Paraná. A outra dissertação, desenvolvida na Universidade do Noroeste do Estado do Rio Grande do Sul, aborda o movimento CTS (Ciência, Tecnologia, Sociedade).

Os demais estudos analisados, referenciados em Fleck, embora tenham caracterizado estilos de pensamento e dinâmicas de implementação e mudança relativas aos focos que investigaram, não tiveram como objetivo, propriamente, realizar uma análise sobre a produção do conhecimento.

Com relação às razões que levaram os pesquisadores a optar pelo aporte epistemológico de Fleck, os estudos destacam, com ênfase, o que essa perspectiva possibilitou:

- identificar o caráter sócio-histórico-cultural da produção do conhecimento (LEITE, 2004; DELIZOICOV, 2002; SCHEID, 2006 e LORENZETTI, 2008);

- identificar e caracterizar modos de conceber e atuar na pesquisa e no ensino, de acordo com uma perspectiva histórica que inclui o tempo presente: as 11 pesquisas analisadas atribuem esse potencial à epistemologia de Fleck;

- compreender a interação dos coletivos de cientistas (DELIZOICOV, 2002; SLONGO, 2004; LEITE, 2004; SCHEID, 2006; BERTONI, 2007; LORENZETTI, 2008 e MUENCHEN, 2010;) e de professores (MARQUES, 2007 e MUENCHEN, 2010) entre si e com outros grupos sociais, explicitando o caráter sociológico da produção e disseminação do conhecimento científico. Assim, as categorias circulação intra e intercoletiva de ideias possibilitaram caracterizar os processos de constituição, disseminação e modificação do conhecimento;

- conhecer o pensamento e as práticas pedagógicas dos professores (DELIZOICOV, 1995; MARQUES, 2007; BERTONI, 2007 e MUENCHEN, 2010).

Observa-se que as categorias "estilo de pensamento", "coletivo de pensamento" e "circulação de ideias" foram utilizadas por todos os estudos analisados. A disseminação e uso dessas categorias epistemológicas para investigar diferentes objetos na área da Educação em Ciências reflete seu potencial.

Partindo do pressuposto que um Coletivo de Pensamento compartilha conhecimentos e práticas, as quais caracterizam o Estilo de Pensamento em vigor, 
foram investigadas referências bibliográficas utilizadas pelas pesquisas analisadas e que abordam a epistemologia de Fleck $(1986 ; 2010)$. Esse dado é relevante na medida em que explicita o compartilhamento de elementos teóricos e metodológicos pela pesquisa em Educação em Ciências.

Identificou-se que o livro La génesis y el desarrollo de un hecho cientifico (FLECK, 1986) foi citado por todos os trabalhos analisados, seguido da introdução que Schãfer e Schnelle (1986) fazem na introdução à obra do autor, a qual também foi citada por todos os estudos. Os textos de Ilana Löwy (1994a, 1994b, 2004), a grande divulgadora do pensamento epistemológico de Fleck, foram citados por sete das onze pesquisas analisadas. Além disso, com três citações, destacam-se os artigos organizados por Cohen e Schnelle (1986) na obra Cognition and Fact.

Além destes, foram identificados outros 116 textos que fazem referência à epistemologia de Fleck (1986; 2010), dos quais, 44 são teses, dissertações e artigos publicados em periódicos e anais/atas de eventos.

A tese de Da Ros (2000) foi citada por sete textos, e as de Cutolo (2001), Leite (2004) e Delizoicov (2002) por seis. Com cinco citações, encontram-se a dissertação pioneira de Delizoicov (1995) e as teses de Lima (2003) e Pfuetzenreiter (2003). As teses de Backes (2000) e Slongo (2004) foram citadas por quatro pesquisas. Por sua vez, os artigos de Leite, Ferrari e Delizoicov (2001) e Delizoicov et al. (2002) foram citados por sete das onze pesquisas analisadas.

Outro dado que chama a atenção é a quantidade de citações, presentes nas dissertações e teses que utilizaram a epistemologia de Fleck, feitas pelos autores das pesquisas analisadas. Os estudos de Lorenzetti (2008) e Muenchen (2010) localizaram a maioria das dissertações e teses desenvolvidas nos programas de pós-graduação. Já os estudos de Lima (2007) e Mezalira (2008) citaram poucos trabalhos envolvendo a epistemologia fleckiana.

Ao analisar as referências compartilhadas na produção acadêmica em Educação Ambiental, Lorenzetti (2008, p. 349) identificou a "[...] incipiente Circulação Intracoletiva de Ideias, principalmente em relação às referências das distintas dissertações e teses de Educação Ambiental desenvolvidas nos programas de Pós-Graduação no Brasil". Argumenta o autor para que "haja maior disseminação dos conhecimentos e práticas que estão sendo vinculadas nestas pesquisas é necessário referenciar e discutir o que já se produziu na área, ampliando as discussões [...]. Uma intensificação no uso mais compartilhado de referências potencializaria a extensão do Estilo de Pensamento" (LORENZETTI, 2008, p. 349). Da mesma forma, conforme destacado, pode-se verificar que alguns autores das pesquisas analisadas não referenciaram trabalhos assemelhados, pouco contribuindo para a disseminação e ampliação da epistemologia de Fleck. Entende-se que estudos que se referiam às pesquisas já realizadas podem contribuir para potencializar novos estudos, balizados pelas contribuições epistemológicas do autor.

$\mathrm{O}$ uso de entrevistas consistentemente estruturadas, articuladas à pesquisa documental, é procedimento que possibilita análises, tendo como referência a perspectiva fleckiana (MUENCHEN, 2010). Os procedimentos metodológicos 
destacados nos trabalhos analisados apontam que as análises documentais e as entrevistas constituem elementos que possibilitam tanto resgates históricos quanto análises que caracterizam circulação de ideias, conhecimentos e práticas entre os círculos esotérico e exotérico.

\section{CONSIDERACְ̃̃ES FINAIS}

O estudo realizado teve o objetivo de identificar em que momento Fleck se estabeleceu como um referencial importante para a pesquisa, especialmente na área de Educação em Ciências e qual a relação que a pesquisa desenvolvida na área estabeleceu com esse referencial. Os dados revelaram que foi em meados da década de 1990 que sugiram os primeiros estudos subsidiados por essa perspectiva epistemológica. Mostram também que há uma concentração de trabalhos em instituições do sul do Brasil, notadamente na Universidade Federal de Santa Catarina, especialmente na área de Educação em Ciências, estando o maior volume de estudos concentrados no eixo "emergência de um fato científico", seguido pelos estudos sobre a "formação de professores" e "análise da produção acadêmica".

Com relação às contribuições da epistemologia de Fleck para a pesquisa na área de Educação em Ciências, houve destaque para as seguintes justificativas: possibilita compreender a constituição de uma área do conhecimento; explicitar o caráter sociológico tanto da produção quanto da disseminação do conhecimento; identificar as condições para a instauração de um estilo de pensamento ligado à ciência; compreender a importância de comunicação intra e intercoletiva no estabelecimento e transformação de um estilo de pensamento; analisar o peso da formação para o ingresso em um estilo de pensamento; entender melhor a relação teoria e prática na formação dos professores; refletir sobre a prática pedagógica dos professores; desenvolver alternativas para a inserção da história da ciência nos currículos da graduação.

O estudo não teve a pretensão de dimensionar o alcance ou o impacto da incorporação dos pressupostos epistemológicos fleckianos na produção científica, mas explicitar o diálogo que a área de Educação em Ciências tem feito com a perspectiva epistemológica de Fleck. Assim, a perspectiva com que foi realizada essa análise visou, sobretudo, apresentar um panorama que possa contribuir para novas reflexões.

\section{REFERÊNCIAS}

AMORIM, M. A. L. Parâmetros curriculares nacionais para o ensino médio e professores de biologia: dificuldades de interlocução. 2004. Tese (Doutorado em Educação) - Universidade Federal de Santa Catarina, Florianópolis - SC, 2004.

ARAÚJO, R. L. C. da C. Doenças construção e realidade na formação dos médicos. Objeto Fronteira como instrumento de interação entre diferentes estilos de pensamento. 2002. Dissertação (Mestrado em Educação) - Universidade Federal de Santa Catarina, Florianópolis - SC, 2002. 
BACKES, V. M. S. Estilo de pensamento e práxis na enfermagem: a contribuição do estágio pré-profissional. 1999. Tese (Doutorado em Enfermagem) - Universidade Federal de Santa Catarina, Florianópolis - SC, 1999.

BARDIN, L. Análise de conteúdo. Portugal: Edições 70, 2008.

BERTONI, D. Um estudo dos estilos de pensamento biológico sobre o fenômeno vida. 2007. Dissertação (Mestrado em Educação) - Universidade Federal do Paraná, Curitiba - PR, 2007.

BISCHOFF, H. F. O estilo de pensamento em medicina estética: reflexões sobre as bases epistemológicas da formação médica. 2009. Dissertação (Mestrado em Educação) - Universidade do Extremo Sul Catarinense, Criciúma - SC, 2009.

CAVALLI, C. Reflexões sobre a educação física no SUS: uma análise a partir das dissertações e teses. 2009. Dissertação (Mestrado em Educação Física) - Universidade Federal de Santa Catarina, Florianópolis - SC, 2009.

COSTA, N. L. Estilos de pensamento em acupuntura: uma análise epistemológica. 2009. Dissertação (Mestrado Profissionalizante em Saúde) - Universidade do Vale do Itajaí, Itajaí -SC, 2009.

CUTOLO, L. R. A. Estilo de pensamento em educação médica um estudo do currículo do curso de graduação em Medicina da UFSC. 2001. Tese (Doutorado em Educação) - Universidade Federal de Santa Catarina, Florianópolis - SC, 2001.

DA ROS, M. A. Estilo de pensamento em educação médica: um estudo da produção da FSP-USP e ENSPFIOCRUZ entre 1948 e 1994, a partir de epistemologia de Ludwik Fleck. 2000. Tese (Doutorado em Educação) - Universidade Federal de Santa Catarina, Florianópolis - SC, 2000.

DELIZOICOV, D. et al. Sociogênese do conhecimento e pesquisa em ensino: contribuições a partir do referencial fleckiano. Caderno Brasileiro do Ensino de Física. Florianópolis, SC, v. 19, número especial, p. 52-69, jun. 2002.

DELIZOICOV, D.; ANGOTTI, J. A.; PERNAMBUCO, M.M. Ensino de Ciências: fundamentos e métodos. São Paulo: Cortez, 2002.

DELIZOICOV, N. C. O professor de ciências naturais e o livro didático. 1995. Dissertação (Mestrado em Educação) - Universidade Federal de Santa Catarina, Florianópolis - SC, 1995.

. O movimento do sangue no corpo bumano: história e ensino. 2002. Tese (Doutorado em Educação) - Universidade Federal de Santa Catarina, Florianópolis - SC, 2002.

FLECK, L. La génesis y el desarrollo de um hecho científico. Madrid: Alianza Editorial, 1986.

Gênese e desenvolvimento de um fato cientifico. Belo Horizonte: Fabrefactum. 2010.

GOMES, D. Etiologia da cárie uma construção do estilo de pensamento. 2002. Dissertação (Mestrado em Saúde Coletiva) - Universidade Federal de Santa Catarina, Florianópolis - SC, 2002.

GUEDES, C. R. A subjetividade como anomalia: estratégias médicas para lidar com os sintomas vagos e difusos em Biomedicina. 2007. Tese (Doutorado em Saúde Coletiva) - Universidade do Estado do Rio de Janeiro, Rio de Janeiro - RJ, 2007.

KOIFMAN, L. A crítica do modelo biomédico na reformulação curricular do curso de Medicina da Universidade Federal Fluminense. 1996. Dissertação (Mestrado em Saúde Pública) - Fundação Oswaldo Cruz, Rio de Janeiro - RJ, 1996.

KOSLOWSKI, A. A. Nas origens da estrutura das revoluções científicas: a influência de Fleck, Polanyi e Quine na filosofia da ciência de Thomas Samuel Kuhn. 2004. Dissertação (Mestrado em Filosofia) - Universidade Federal de Santa Catarina, Florianópolis - SC, 2004.

LAMBACH, M. Atuação e formação dos professores de química na EJA: características dos estilos de pensamento - um olhar a partir de Fleck. 2007. Dissertação (Mestrado em Educação Científica e Tecnológica) - Universidade Federal de Santa Catarina, Florianópolis - SC, 2007.

LEITE, R. C. M. A produção coletiva do conbecimento científico: um exemplo no ensino de genética. 2004. Tese (Doutorado em Educação) - Universidade Federal de Santa Catarina, Florianópolis - SC, 2004.

LEITE, R. C. M.; FERRARI, N; DELIZOICOV, D. A história das leis de Mendel na perspectiva fleckiana. Revista Brasileira de Pesquisa em Educação em Ciências, São Paulo-SP, v.1, n.2, p. 97-108, maio/ago. 2001.

LIMA, A. M. C. Estilo de pensar no ensino de medicina homeopática. 2003. Tese (Doutorado em Educação) - Universidade Federal de Santa Catarina, Florianópolis - SC, 2003. 
LIMA, L. C. de. A formação dos professores de ciências: uma abordagem epistemológica. 1999. Dissertação (Mestrado em Educação) - Universidade Federal de Santa Catarina, Florianópolis - SC, 1999.

Processo de planejamento e implantação do Parque Natural Municipal de Lages-SC com ênfase na conservação de bacias hidrográficas e na percepção da comunidade do entorno. 2007. Tese (Doutorado em Engenharia Ambiental) - Universidade Federal de Santa Catarina, Florianópolis - SC, 2007.

LORENZETTI, L. Estilos de pensamento em educação ambiental: uma análise a partir das dissertações e teses. 2008. Tese (Doutorado em Educação Científica e Tecnológica) - Universidade Federal de Santa Catarina, Florianópolis - SC, 2008.

LÖWY, I. Ludwik Fleck e a presente história das ciências. In: MANGUINHOS - HISTÓRIA, CIÊNCIAS, SAÚDE. Rio de Janeiro: Fiocruz, v. 1, n. 1, 1994a.

. Fleck e a historiografia recente da pesquisa biomédica. In: PORTOCARRERO, V. (Org.) Filosofia, história e sociologia das ciências: abordagens contemporâneas. Rio de Janeiro: Fiocruz, 1994b. . Introduction: Ludwik Fleck's epistemology of medicine and biomedical sciences. Stud. Hist. Phil. Biol \& Biomed. Sc., n. 35, p. 437-445, 2004.

LÜDKE, L. Formaşão de docentes para o SUS: um desafio sanitário e pedagógico. 2009. Dissertação (Mestrado Profissionalizante em Saúde) - Universidade do Vale do Itajaí, Itajaí - SC, 2009.

MACHADO, N. H. S. O ensinar e o aprender a fažer pesquisa. O real e o desejado. 2008. Dissertação (Mestrado em Educação) - Universidade de Brasília, Brasília - DF, 2008.

MAEYAMA, M. A. Estilos de pensamento em odontologia social e preventiva: um estudo da disciplina de odontologia social e preventiva do curso de odontologia da UNIVALI. 2006. Dissertação (Mestrado Profissionalizante em Saúde) - Universidade do Vale do Itajaí, Itajaí - SC, 2006.

MEZALIRA, S. M. Enfoque CTS no ensino de ciências naturais a partir de publicações em eventos científicos no Brasil. 2008. Dissertação (Mestrado em Educação nas Ciências) - Universidade Regional do Noroeste do Estado do Rio Grande do Sul, Ijuí - RS, 2008.

MUENCHEN, C. Disseminação dos três momentospedagógicos: um estudo sobre práticas docentes na região de Santa Maria - RS. 2010. (Doutorado em Educação Científica e Tecnológica) - Universidade Federal de Santa Catarina, Florianópolis - SC, 2010.

NEDER, C. J. Comunidade científica e natureza no pensamento de T. S. Kuhn. 2001. Dissertação (Mestrado em Filosofia) - Universidade do Estado do Rio de Janeiro, Rio de Janeiro - RJ, 2001.

NOGUEIRA, M. I. Entre a conversão e o ecletismo: de como médicos brasileiros tornam-se "chineses". 2003. Tese (Doutorado em Saúde Coletiva) - Universidade do Estado do Rio de Janeiro, Rio de Janeiro - RJ, 2003.

PARREIRAS, M. M. M. Ludwik Fleck e a historiografia da ciência: diagnóstico de um estilo de pensamento segundo as ciências da vida. 2006. Dissertação (Mestrado em História) - Universidade Federal de Minas Gerais, Belo Horizonte - MG, 2006.

PFUETZENREITER, M. R. O ensino da medicina veterinária preventiva e saúde pública nos cursos de medicina veterinária: estudo de caso realizado na Universidade do Estado de Santa Catarina. 2003. Tese (Doutorado em Educação) - Universidade Federal de Santa Catarina, Florianópolis - SC, 2003.

QUARESMA, S. J. L. A percep̧cão do médico clínico em relação aos pacientes hipocondríacos e poliqueixosos que são atendidos no ambulatório do Hospital Universitário Professor Polydoro Ernani de São Thiago. 2005. Dissertação. (Mestrado em Sociologia Política) - Universidade Federal de Santa Catarina, Florianópolis - SC, 2005.

QUEIRÓS, E. P. de; NARDI, R. Um panorama da epistemologia de Ludwik Fleck na pesquisa em ensino de ciências. In: ENCONTRO DE PESQUISA EM ENSINO DE FÍSICA, 11., 2008, Curitiba. Anais... Curitiba: SBF, 2008. p. 1-11.

SALLES, A. C. de. Nem gênios, nem heróis: a história da ciência em Ludwik Fleck. 2007. Dissertação (Mestrado em História) - Universidade Federal de Minas Gerais, Belo Horizonte - MG, 2007.

SANTOS, A. L. G. dos. Uma Construção dos saberes sobre a epidemia de AIDS - os formulários de notificação de casos em perspectiva (1982-98). 1999. Dissertação (Mestrado em Saúde Pública) Fundação Oswaldo Cruz, Rio de Janeiro - RJ, 1999.

SANTOS, M. A. M. As diretrizes curriculares e o currículo de graduação em medicina da UNIVALI: construindo a interdisciplinaridade através dos objetos fronteiriços e da epistemologia de Fleck. 
2005. Dissertação (Mestrado Profissionalizante em Saúde) - Universidade do Vale do Itajaí, Itajaí - SC, 2005.

SCHÄFER, L.; SCHNELLE, T. Los fundamentos de la visión sociológica de Ludwik Fleck de la teoria de la ciência. In: FLECK, L. La gênesis y el desarrollo de um hecho científico. Madrid: Alianza Editorial, 1986.

SCHEID, N. M. J. A contribuição da história da biologia na formação inicial de professores de ciências biológicas. 2006. Orientador: Nadir Ferrari. 2006. 215 f. Tese (Doutorado em Educação Científica e Tecnológica) - Universidade Federal de Santa Catarina, Florianópolis - SC, 2006.

SILVA, W. B. da. A emergência da atenção farmacêutica: um olhar epistemológico e contribuições para o seu ensino. Orientador: Demétrio Delizoicov Neto. 2009. 305 f. Tese (Doutorado em Educação Científica e Tecnológica) - Universidade Federal de Santa Catarina, Florianópolis - SC, 2009.

SKALINSKI, L. M. Epidemiologia e epidemiologia crítica: considerações sobre diferentes estilos de pensamento. 2008. 141 f. Dissertação (Mestrado em Saúde Pública) - Universidade Federal de Santa Catarina, Florianópolis - SC, 2008.

SLONGO, Iône. Inês. Pinsson. A produção acadêmica em ensino de biologia: um estudo a partir de teses e dissertações. Orientador: Demétrio Delizoicov Neto. 2004. 364 f. Tese (Doutorado em Educação) - Universidade Federal de Santa Catarina, Florianópolis - SC, 2004.

TAVARES, A. L. C. O ensino de patologia bumana e suas relações históricas com o estilo de pensamento a partir da análise de livros-texto. 2008. 112 f. Dissertação (Mestrado em Educação) - Universidade de Brasília, Brasília - DF, 2008.

TESSER, Charles Dalcanale. Epistemologia contemporânea e saúde: a luta pela verdade e as práticas terapêuticas. Orientador: Gastão W. de S. Campos 2004. 392 f. Tese (Doutorado em Saúde Coletiva) - Universidade Estadual de Campinas, Campinas - SP, 2004.

UCHÔA, Severina Alice da Costa. Osprotocolos e a decisão médica: evidências e ou vivências?. Orientador: Kenneth Rochel de Camargo. 2003. 135 f. Tese (Doutorado em Saúde Coletiva) - Universidade do Estado do Rio de Janeiro, Rio de Janeiro - RJ, 2003. 